\title{
Dihadron Azimuthal Correlation from Collins Effect in Unpolarized Hadron Collisions
}

\author{
Zhong-Bo Kang ${ }^{1, *}$ and Feng Yuan ${ }^{1,2, \dagger}$ \\ ${ }^{1}$ RIKEN BNL Research Center, Brookhaven National Laboratory, Upton, NY 11973, USA \\ ${ }^{2}$ Nuclear Science Division, Lawrence Berkeley \\ National Laboratory, Berkeley, CA 94720, USA
}

(Dated: October 24, 2018)

\begin{abstract}
We study the dihadron azimuthal correlation produced nearly back-to-back in unpolarized hadron collisions, arising from the product of two Collins fragmentation functions. Using the latest Collins fragmentation functions extracted from the global analysis of available experimental data, we make predictions for the azimuthal correlation of two-pion production in $p p$ collisions at RHIC energies. We find that the correlation is sizable in the mid-rapidity region for moderate jet transverse momentum.
\end{abstract}

PACS numbers: 12.38.Bx, 12.39.St, 13.85.Qk, 13.88+e

*Electronic address: zkang@bnl.gov

†Electronic address: fyuan@lbl.gov 


\section{INTRODUCTION}

The transverse momentum dependent (TMD) distributions and fragmentation functions have received much attention recently [1]. They are believed to be responsible for several azimuthal asymmetries observed in experiments, such as the single transverse spin asymmetry (SSA) in semi-inclusive hadron production in deep inelastic scattering (SIDIS) [2, 3] and in hadronic collisions [4], as well as the large $\cos (2 \phi)$ anomalous azimuthal asymmetry in back-to-back dihadron production in $e^{+} e^{-}$annihilation [5].

Among these TMD parton distributions and fragmentation functions, the Sivers quark distribution [6] and the Collins fragmentation function [7] are mostly discussed in the last few years. The Sivers quark distribution represents a distribution of unpolarized quarks in a transversely polarized nucleon, through a correlation between the quark's transverse momentum and the nucleon polarization vector. On the other hand, the Collins fragmentation function describes a transversely polarized quark jet fragmenting into an unpolarized hadron, whose transverse momentum relative to the jet axis correlates with the transverse polarization vector of the fragmenting quark.

Though both of them belong to the so-called "naive-time-reversal-odd" (T-odd) functions, they have very different universality properties. For the Sivers functions, it has been shown that they differ by a sign for the SIDIS and Drell-Yan (DY) processes [8], and those in the hadronic collisions have even more nontrivial relation to that in SIDIS and DY processes [9 13]. On the other hand, the Collins fragmentation function is universal between different processes, in the SIDIS, $e^{+} e^{-}$and hadronic collisions [14 16]. The effect of the Collins fragmentation function has been recently explored by one of us in the azimuthal asymmetric distribution of hadrons inside a jet in $p^{\uparrow} p$ collision [17]. It is demonstrated that the asymmetry is sizable at RHIC, therefore, the experimental study of this process could provide an important information on the universality of the Collins fragmentation function.

Another difference between these two functions is that the Collins fragmentation function is chiral-odd whereas the Sivers function is chiral-even. Because of its chiral-odd nature, the Collins effect can only be observed when it is coupled to another chiral-odd distribution or fragmentation function. In SIDIS, the chiral-odd quark transversity [18] can couple to the Collins fragmentation function and leads to nonzero azimuthal SSA [7]. This SSA has been studied by the HERMES [2] and COMPASS [3] collaborations, and very interesting 
results on the Collins fragmentation function have been found. In $e^{+} e^{-}$annihilation process, two Collins fragmentation functions couple to each other in the back-to-back dihadron

production, results into to a $\cos (2 \phi)$ azimuthal asymmetry [19]. This anomalous $\cos (2 \phi)$ asymmetry has been measured by the BELLE Collaboration [5], and was found consistent with the HERMES and COMPASS measurements on the Collins fragmentation functions. Recently a global analysis of these experimental data has been performed and the Collins fragmentation functions have been extracted [20].

In this paper we investigate the possibility of exploring the Collins fragmentation function in unpolarized $p p$ collision by studying the azimuthal correlation in back-to-back dihadron production, following the same wisdom of dihadron production in $e^{+} e^{-}$annihilation. We show that the asymmetry is proportional to the product of two Collins fragmentation function, same as that in $e^{+} e^{-}$annihilation. Using the latest Collins fragmentation function extracted from the global analysis of available data on SIDIS and $e^{+} e^{-}$experiments, we estimate the asymmetry for dihadron production at RHIC energy. We find that the azimuthal asymmetry is sizable at mid-rapidity region for moderate jet transverse momentum. We argue that this process shall provide addtional important information on the Collins fragmentation function and its universality properties.

The rest of the paper is organized as follows. In Sec. III, we derive the theoretical results for the dihadron azimuthal correlation produced nearly back-to-back in unpolarized hadron collision. In Sec. III, we present our numerical predictions for the azimuthal correlation in unpolarized $p p$ collisions for RHIC kinematics. Finally, we summarize our findings and the corresponding conclusions in Sec. IV.

\section{DIHADRON AZIMUTHAL CORRELATION IN UNPOLARIZED HADRON COLLISION}

We study the azimuthal correlation of two hadrons $h_{1}$ and $h_{2}$ produced nearly back-toback in a hadronic collision,

$$
A\left(P_{1}\right)+B\left(P_{2}\right) \rightarrow h_{1}\left(P_{h 1}\right)+h_{2}\left(P_{h 2}\right)+X
$$

where both of the incident hadrons $A$ and $B$ are unpolarized. The momenta of the initial hadrons are denoted by $P_{1}$ and $P_{2}$, and those of the final hadrons by $P_{h 1}$ and $P_{h 2}$, respectively. 


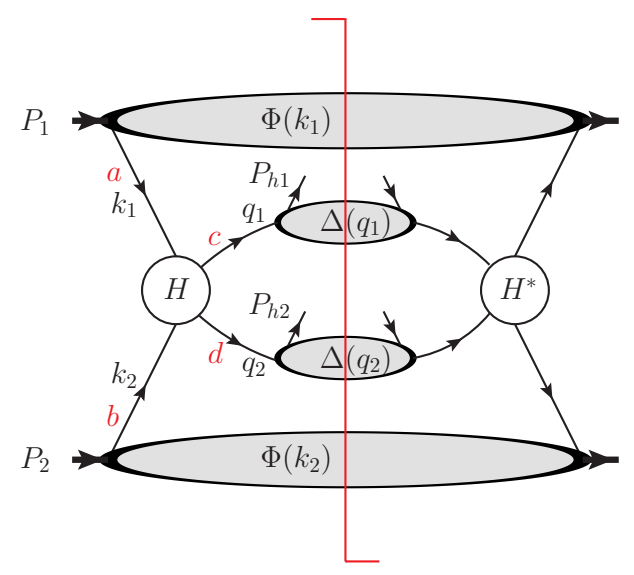

FIG. 1: The leading order contribution to the cross section of $A\left(P_{1}\right)+B\left(P_{2}\right) \rightarrow h_{1}\left(P_{h 1}\right)+h_{2}\left(P_{h 2}\right)+$ $X$ with the $2 \rightarrow 2$ partonic process $a\left(k_{1}\right)+b\left(k_{2}\right) \rightarrow c\left(q_{1}\right)+d\left(q_{2}\right)$.

The leading order contribution to the scattering cross section comes from partonic $2 \rightarrow 2$ sub-processes, $a\left(k_{1}\right)+b\left(k_{2}\right) \rightarrow c\left(q_{1}\right)+d\left(q_{2}\right)$, as shown in Fig. 1. The parton momenta are expanded as follows,

$$
\begin{gathered}
k_{1}=x_{1} P_{1}+k_{1 T}, \\
k_{2}=x_{2} P_{2}+k_{2 T}, \\
P_{h 1}=z_{1} q_{1}+p_{1 T}, \\
P_{h 2}=z_{2} q_{2}+p_{2 T},
\end{gathered}
$$

where $x_{1}$ and $x_{2}$ are the longitudinal momentum fractions, and $k_{1 T}$ and $k_{2 T}$ are the transverse momentum of the parton relative to the corresponding incident hadron. $q_{1}$ and $q_{2}$ are the momenta of the nearly back-to-back jets $J_{1}$ and $J_{2}$, which has a polar angle $\theta_{1}$ and $\theta_{2}$ relative to the incoming hadron $P_{1}$, respectively. The momenta of the incoming hadrons and the final state two jets form the so-called reaction plane (approximately). Besides carrying a longitudinal momentum fraction $z_{1}\left(z_{2}\right)$ of the jet $J_{1}\left(J_{2}\right)$, the hadron $h_{1}\left(h_{2}\right)$ also has a transverse momentum $p_{1 T}\left(p_{2 T}\right)$ relative to the jet $J_{1}\left(J_{2}\right)$ direction, which defines an azimuthal angle with the reaction plane: $\phi_{1}\left(\phi_{2}\right)$, as shown in Fig. 2. Due to the Collins effect, there will be a correlation between these two azimuthal angles $\phi_{1}$ and $\phi_{2}$, which is proportional to the product of the two Collins fragmentation functions as we will show below. 


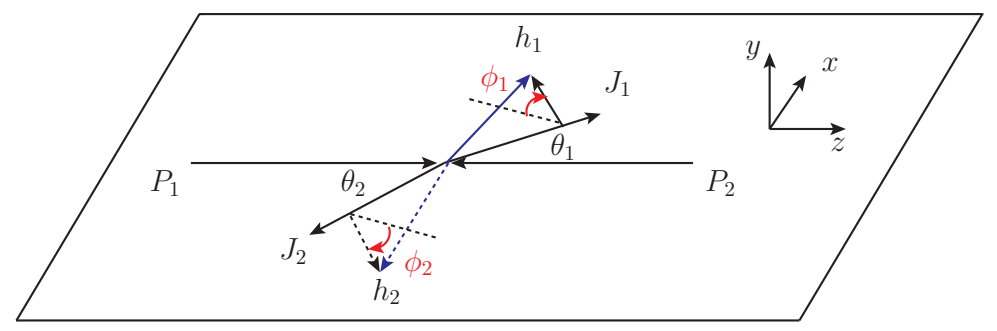

FIG. 2: Illustration of the kinematics for dihadron production $A B \rightarrow h_{1} h_{2}+X$.

From Fig. 1, the cross section of dihadron production can be written as [13],

$$
\begin{aligned}
d \sigma= & \frac{1}{2 S} \int d x_{1} d^{2} k_{1 T} d x_{2} d^{2} k_{2 T} d z_{1} d^{2} p_{1 T} d z_{2} d^{2} p_{2 T} \frac{d^{3} q_{1}}{(2 \pi)^{3} 2 E_{1}} \frac{d^{3} q_{2}}{(2 \pi)^{3} 2 E_{2}}(2 \pi)^{4} \delta^{4}\left(k_{1}+k_{2}-q_{1}-q_{2}\right) \\
& \times \operatorname{Tr}\left[\Phi\left(x_{1}, k_{1 T}\right) \Phi\left(x_{2}, k_{2 T}\right) \Delta\left(z_{1}, p_{1 T}\right) \Delta\left(z_{2}, p_{2 T}\right) H\left(k_{1}, k_{2}, q_{1}, q_{2}\right) H^{*}\left(k_{1}, k_{2}, q_{1}, q_{2}\right)\right], \quad
\end{aligned}
$$

where $S=\left(P_{1}+P_{2}\right)^{2}$, and $\Phi\left(x, k_{T}\right)$ and $\Delta\left(z, p_{T}\right)$ are the distribution and fragmentation correlation functions, and $H\left(k_{1}, k_{2}, q_{1}, q_{2}\right)$ are the hard part amplitude.

For the unpolarized hadron, the correlation functions $\Phi\left(x, k_{T}\right)$ can be simply decomposed as [21, 22],

$$
\Phi\left(P, x, k_{T}\right)=\frac{1}{2}\left[f\left(z, k_{T}^{2}\right) \not P+h_{1}^{\perp}\left(z, k_{T}^{2}\right) \frac{\sigma^{\mu \nu} k_{T \mu} P_{\nu}}{M}\right]
$$

where $f\left(x, k_{T}^{2}\right)$ is the unpolarized TMD parton distribution function, and $h_{1}^{\perp}\left(z, k_{T}^{2}\right)$ is the Boer-Mulders function [22]. Similarly, we can parameterize the gluon distributions from the incoming hadrons. The effect of Boer-Mulders function in unpolarized hadronic collisions has been extensively studied previously [23 25], which will be neglected in our current study. We will concentrate on the effect coming from the fragmentation correlation function $\Delta\left(z, p_{T}\right)$, which can be expanded as [26]

$$
\Delta\left(P_{h}, z, p_{T}\right)=\frac{1}{2}\left[D\left(z, p_{T}^{2}\right) P_{h}+H_{1}^{\perp}\left(z, p_{T}^{2}\right) \frac{\sigma^{\mu \nu} P_{h \mu} p_{T \nu}}{z M_{h}}\right],
$$

where $D\left(z, p_{T}^{2}\right)$ is the unpolarized TMD fragmentation function, and $H_{1}^{\perp}$ is the Collins function. From its definition, we can see that the Collins function describes a transversely polarized quark jet fragmenting into an unpolarized hadron [7]. It is this Collins function that generates a non-vanishing azimuthal correlation between the final state two hadrons. Since Collins function is a chiral-odd TMD function, the azimuthal correlation will depend on the product of two Collins functions. 
The phase space integral in Eq. (3) can be simplified by using

$$
\begin{aligned}
& \frac{d^{3} q_{1}}{(2 \pi)^{3} 2 E_{1}} \frac{d^{3} q_{2}}{(2 \pi)^{3} 2 E_{2}}(2 \pi)^{4} \delta^{4}\left(k_{1}+k_{2}-q_{1}-q_{2}\right) \\
& =d y_{1} d y_{2} d P_{\perp}^{2}\left(\frac{1}{8 \pi S}\right) \delta\left[x_{1}-\frac{P_{\perp}}{\sqrt{S}}\left(e^{y_{1}}+e^{y_{2}}\right)\right] \delta\left[x_{2}-\frac{P_{\perp}}{\sqrt{S}}\left(e^{-y_{1}}+e^{-y_{2}}\right)\right],
\end{aligned}
$$

where $y_{1}$ and $y_{2}$ are rapidities for the jet $J_{1}$ and $J_{2}, P_{\perp}$ is the jet transverse momentum. Since $p_{1 T}, p_{2 T} \ll P_{\perp}$, the rapidity of the hadron is approximately equal to that of the parent jet. As stated above, we neglect the intrinsic transverse momentum effects for the incoming partons. Finally we obtain the cross section for this process as

$$
\begin{aligned}
\frac{d \sigma}{d \mathcal{P S}}= & \frac{\pi \alpha_{s}^{2}}{S^{2}} \sum_{a b c d} \frac{f_{a}\left(x_{1}\right)}{x_{1}} \frac{f_{b}\left(x_{2}\right)}{x_{2}}\left[D_{c \rightarrow h_{1}}\left(z_{1}, p_{1 T}^{2}\right) D_{d \rightarrow h_{2}}\left(z_{2}, p_{2 T}^{2}\right) H_{a b \rightarrow c d}^{U}(\hat{s}, \hat{t}, \hat{u})\right. \\
& +\left(p_{1 T} \cdot p_{2 T}-\frac{x_{1} x_{2}}{P_{\perp}^{2}}\left(P_{1} \cdot p_{1 T} P_{2} \cdot p_{2 T}+P_{1} \cdot p_{2 T} P_{2} \cdot p_{1 T}\right)\right) \\
& \left.\times \frac{H_{1}^{\perp}\left(z_{1}, p_{1 T}^{2}\right)}{z_{1} M_{h}} \frac{H_{1}^{\perp}\left(z_{2}, p_{2 T}^{2}\right)}{z_{2} M_{h}} H_{a b \rightarrow c d}^{\text {Collins }}(\hat{s}, \hat{t}, \hat{u})\right],
\end{aligned}
$$

where $d \mathcal{P} \mathcal{S} \equiv d y_{1} d y_{2} d P_{\perp}^{2} d z_{1} d z_{2} d^{2} p_{1 T} d^{2} p_{2 T}$ is the phase space for this process, $f_{a}\left(x_{1}\right)$ and $f_{b}\left(x_{2}\right)$ are the standard unpolarized parton distribution functions, and $\hat{s}, \hat{t}$, and $\hat{u}$ are the usual partonic Mandelstam variables. The parton momentum fractions $x_{1}$ and $x_{2}$ are fixed by the delta functions in Eq. (6),

$$
\begin{aligned}
& x_{1}=\frac{P_{\perp}}{\sqrt{S}}\left(e^{y_{1}}+e^{y_{2}}\right), \\
& x_{2}=\frac{P_{\perp}}{\sqrt{S}}\left(e^{-y_{1}}+e^{-y_{2}}\right) .
\end{aligned}
$$


The normal partonic cross sections $H_{a b \rightarrow c d}^{U}$ are well-known [27],

$$
\begin{aligned}
H_{g g \rightarrow q \bar{q}}^{U} & =\frac{1}{2 N_{c}}\left[\frac{\hat{t}}{\hat{u}}+\frac{\hat{u}}{\hat{t}}\right]-\frac{N_{c}}{N_{c}^{2}-1}\left[\frac{\hat{t}^{2}+\hat{u}^{2}}{\hat{s}^{2}}\right], \\
H_{q \bar{q} \rightarrow q^{\prime} \bar{q}^{\prime}}^{U} & =\frac{N_{c}^{2}-1}{2 N_{c}^{2}}\left[\frac{\hat{t}^{2}+\hat{u}^{2}}{\hat{s}^{2}}\right], \\
H_{q \bar{q} \rightarrow q \bar{q}}^{U} & =\frac{N_{c}^{2}-1}{2 N_{c}^{2}}\left[\frac{\hat{t}^{2}+\hat{u}^{2}}{\hat{s}^{2}}+\frac{\hat{s}^{2}+\hat{u}^{2}}{\hat{t}^{2}}\right]-\frac{N_{c}^{2}-1}{N_{c}^{3}}\left[\frac{\hat{u}^{2}}{\hat{s} \hat{t}}\right], \\
H_{q q^{\prime} \rightarrow q q^{\prime}}^{U} & =\frac{N_{c}^{2}-1}{2 N_{c}^{2}}\left[\frac{\hat{s}^{2}+\hat{u}^{2}}{\hat{t}^{2}}\right], \\
H_{q q \rightarrow q q}^{U} & =\frac{N_{c}^{2}-1}{2 N_{c}^{2}}\left[\frac{\hat{s}^{2}+\hat{u}^{2}}{\hat{t}^{2}}+\frac{\hat{s}^{2}+\hat{t}^{2}}{\hat{u}^{2}}\right]-\frac{N_{c}^{2}-1}{N_{c}^{3}}\left[\frac{\hat{s}^{2}}{\hat{t} \hat{u}}\right], \\
H_{q \bar{q} \rightarrow g g}^{U} & =\frac{4\left(N_{c}^{2}-1\right)}{N_{c}^{3}}\left[\frac{\hat{t}}{\hat{u}}+\frac{\hat{u}}{\hat{t}}\right]-\frac{N_{c}^{2}-1}{N_{c}}\left[\frac{\hat{t}^{2}+\hat{u}^{2}}{\hat{s}^{2}}\right] \\
H_{g q \rightarrow g q}^{U} & =-\frac{N_{c}^{2}-1}{2 N_{c}^{2}}\left[\frac{\hat{s}}{\hat{u}}+\frac{\hat{u}}{\hat{s}}\right]+\left[\frac{\hat{s}^{2}+\hat{u}^{2}}{\hat{t}^{2}}\right] \\
H_{g g \rightarrow g g}^{U} & =\frac{4 N_{c}^{2}}{N_{c}^{2}-1}\left[3-\frac{\hat{t} \hat{u}}{\hat{s}^{2}}-\frac{\hat{s} \hat{u}}{\hat{t}^{2}}-\frac{\hat{s}_{\hat{t}}}{\hat{u}^{2}}\right]
\end{aligned}
$$

The new hard parts $H_{a b \rightarrow c d}^{\text {Collins }}$ that are responsible for the azimuthal correlation are given by,

$$
\begin{aligned}
H_{g g \rightarrow q \bar{q}}^{\text {Collins }} & =\frac{1}{N_{c}}-\frac{N_{c}}{N_{c}^{2}-1}\left[\frac{2 \hat{t} \hat{u}}{\hat{s}^{2}}\right], \\
H_{q \bar{q} \rightarrow q^{\prime} \bar{q}^{\prime}}^{\text {Collins }} & =\frac{N_{c}^{2}-1}{N_{c}^{2}}\left[\frac{\hat{t} \hat{u}}{\hat{s}^{2}}\right], \\
H_{q \bar{q} \rightarrow q \bar{q}}^{\text {Collins }} & =\frac{N_{c}^{2}-1}{N_{c}^{2}}\left[\frac{\hat{t} \hat{u}}{\hat{s}^{2}}\right]-\frac{N_{c}^{2}-1}{N_{c}^{3}}\left[\frac{\hat{u}}{\hat{s}}\right], \\
H_{q q^{\prime} \rightarrow q q^{\prime}}^{\text {Collins }} & =0 \\
H_{q q \rightarrow q q}^{\text {Collins }} & =\frac{N_{c}^{2}-1}{N_{c}^{3}},
\end{aligned}
$$

and the hard parts for the channels with gluon in the final state, $q \bar{q} \rightarrow g g, q g \rightarrow q g$ and $g g \rightarrow g g$ vanish since there is no gluon Collins function.

\section{PHENOMENOLOGY STUDY}

In this section we first properly define the azimuthal asymmetry to be measured in the experiments. We then use the latest Collins fragmentation function to estimate this asymmetry for RHIC kinematics. 
From Eq. (7), the explicit form of the azimuthal correlation depends on the following function,

$$
h\left(p_{1 T}, p_{2 T}, \phi_{1}, \phi_{2}\right) \equiv p_{1 T} \cdot p_{2 T}-\frac{x_{1} x_{2}}{P_{\perp}^{2}}\left(P_{1} \cdot p_{1 T} P_{2} \cdot p_{2 T}+P_{1} \cdot p_{2 T} P_{2} \cdot p_{1 T}\right)
$$

Function $h\left(p_{1 T}, p_{2 T}, \phi_{1}, \phi_{2}\right)$ is reduced to its simplest form in the partonic center of mass frame,

$$
h\left(p_{1 T}, p_{2 T}, \phi_{1}, \phi_{2}\right)=-p_{1 T} p_{2 T} \cos \left(\phi_{1}+\phi_{2}\right)
$$

To take advantage of this simplicity, one could boost from the Lab frame to the partonic CM frame experimentally. Or equivalently, one can select the events with $y_{1}+y_{2} \approx 0$, where the Lab frame coincides with the partonic CM frame. In the rest of our paper, we will take $y_{1}+y_{2}=0$ in our calculations and present the numerical estimate for the azimuthal asymmetry.

Integrating over the intrinsic transverse momentum $p_{1 T}$ and $p_{2 T}$, we have

$$
\frac{d \sigma}{d y_{1} d y_{2} d P_{\perp}^{2} d z_{1} d z_{2}}=\frac{\pi \alpha_{s}^{2}}{S^{2}} \sum_{a b c d} \frac{f_{a}\left(x_{1}\right)}{x_{1}} \frac{f_{b}\left(x_{2}\right)}{x_{2}} D_{c \rightarrow h_{1}}\left(z_{1}\right) D_{d \rightarrow h_{2}}\left(z_{2}\right) H_{a b \rightarrow c d}^{U}(\hat{s}, \hat{t}, \hat{u})
$$

While integrating over the moduli of the intrinsic momenta $p_{1 T}$ and $p_{2 T}$, and over the azimuthal angle $\phi_{1}$, one obtain

$$
\begin{aligned}
\frac{d \sigma}{d y_{1} d y_{2} d P_{\perp}^{2} d z_{1} d z_{2} d\left(\phi_{1}+\phi_{2}\right)}= & \frac{\alpha_{s}^{2}}{2 S^{2}} \sum_{a b c d} \frac{f_{a}\left(x_{1}\right)}{x_{1}} \frac{f_{b}\left(x_{2}\right)}{x_{2}}\left[D_{c \rightarrow h_{1}}\left(z_{1}\right) D_{d \rightarrow h_{2}}\left(z_{2}\right) H_{a b \rightarrow c d}^{U}(\hat{s}, \hat{t}, \hat{u})\right. \\
& \left.-\cos \left(\phi_{1}+\phi_{2}\right) \delta \hat{q}_{c \rightarrow h_{1}}^{(1 / 2)}\left(z_{1}\right) \delta \hat{q}_{d \rightarrow h_{2}}^{(1 / 2)}\left(z_{2}\right) H_{a b \rightarrow c d}^{\text {Collins }}(\hat{s}, \hat{t}, \hat{u})\right], \quad(25)
\end{aligned}
$$

where $\delta \hat{q}^{(1 / 2)}(z)$ is the so-called half-moment of the Collins function given by,

$$
\delta \hat{q}^{(1 / 2)}(z)=\int d^{2} p_{T} p_{T} \frac{H_{1}^{\perp}\left(z, p_{T}^{2}\right)}{z M_{h}} .
$$

Then following the normal analysis in $e^{+} e^{-} \rightarrow h_{1} h_{2}+X$, we define

$$
\begin{aligned}
A^{h_{1} h_{2}} & \left(y_{1}, y_{2}, P_{\perp}, z_{1}, z_{2}, \phi_{1}+\phi_{2}\right) \\
& \equiv \frac{d \sigma}{d y_{1} d y_{2} d P_{\perp}^{2} d z_{1} d z_{2} d\left(\phi_{1}+\phi_{2}\right)} / \frac{1}{2 \pi} \frac{d \sigma}{d y_{1} d y_{2} d P_{\perp}^{2} d z_{1} d z_{2}} \\
& =1-\cos \left(\phi_{1}+\phi_{2}\right) \frac{\sum_{a b c d} f_{a}\left(x_{1}\right) f_{b}\left(x_{2}\right) \delta \hat{q}_{c \rightarrow h_{1}}^{(1 / 2)}\left(z_{1}\right) \delta \hat{q}_{d \rightarrow h_{2}}^{(1 / 2)}\left(z_{2}\right) H_{a b \rightarrow c d}^{\text {Collins }}}{\sum_{a b c d} f_{a}\left(x_{1}\right) f_{b}\left(x_{2}\right) D_{c \rightarrow h_{1}}\left(z_{1}\right) D_{d \rightarrow h_{2}}\left(z_{2}\right) H_{a b \rightarrow c d}^{U}}
\end{aligned}
$$


Again to eliminate the false asymmetries [5], we introduce the ratio of the unlike-sign to like-sign pion production, $A_{U}$ and $A_{L}$, given by

$$
\begin{aligned}
R & \equiv \frac{A_{U}}{A_{L}}=\frac{1-\cos \left(\phi_{1}+\phi_{2}\right) P_{U}}{1-\cos \left(\phi_{1}+\phi_{2}\right) P_{L}} \approx 1-\cos \left(\phi_{1}+\phi_{2}\right)\left(P_{U}-P_{L}\right) \\
& \equiv 1-\cos \left(\phi_{1}+\phi_{2}\right) A_{12}\left(y_{1}, y_{2}, P_{\perp}, z_{1}, z_{2}\right)
\end{aligned}
$$

with

$$
\begin{aligned}
& P_{U}=\frac{\sum_{a b c d} f_{a}\left(x_{1}\right) f_{b}\left(x_{2}\right)\left[\delta \hat{q}_{c \rightarrow \pi^{+}}^{(1 / 2)}\left(z_{1}\right) \delta \hat{q}_{d \rightarrow \pi^{-}}^{(1 / 2)}\left(z_{2}\right)+\delta \hat{q}_{c \rightarrow \pi^{-}}^{(1 / 2)}\left(z_{1}\right) \delta \hat{q}_{d \rightarrow \pi^{+}}^{(1 / 2)}\left(z_{2}\right)\right] H_{a b \rightarrow c d}^{\text {Collins }}}{\sum_{a b c d} f_{a}\left(x_{1}\right) f_{b}\left(x_{2}\right)\left[D_{c \rightarrow \pi^{+}}\left(z_{1}\right) D_{d \rightarrow \pi^{-}}\left(z_{2}\right)+D_{c \rightarrow \pi^{-}}\left(z_{1}\right) D_{d \rightarrow \pi^{+}}\left(z_{2}\right)\right] H_{a b \rightarrow c d}^{U}} \\
& P_{L}=\frac{\sum_{a b c d} f_{a}\left(x_{1}\right) f_{b}\left(x_{2}\right)\left[\delta \hat{q}_{c \rightarrow \pi^{+}}^{(1 / 2)}\left(z_{1}\right) \delta \hat{q}_{d \rightarrow \pi^{+}}^{(1 / 2)}\left(z_{2}\right)+\delta \hat{q}_{c \rightarrow \pi^{-}}^{(1 / 2)}\left(z_{1}\right) \delta \hat{q}_{d \rightarrow \pi^{-}}^{(1 / 2)}\left(z_{2}\right)\right] H_{a b \rightarrow c d}^{\text {Collins }}}{\sum_{a b c d} f_{a}\left(x_{1}\right) f_{b}\left(x_{2}\right)\left[D_{c \rightarrow \pi^{+}}\left(z_{1}\right) D_{d \rightarrow \pi^{+}}\left(z_{2}\right)+D_{c \rightarrow \pi^{-}}\left(z_{1}\right) D_{d \rightarrow \pi^{-}}\left(z_{2}\right)\right] H_{a b \rightarrow c d}^{U}} \\
& A_{12}\left(y_{1}, y_{2}, P_{\perp}, z_{1}, z_{2}\right)=P_{U}-P_{L}
\end{aligned}
$$

This way the true asymmetry due to Collins effect is encoded in the so-called double ratio asymmetry parameter $A_{12}\left(y_{1}, y_{2}, P_{\perp}, z_{1}, z_{2}\right)$. To evaluate $A_{12}$ for the dihadron production in unpolarized $p p$ collision at $\sqrt{S}=200 \mathrm{GeV}$ at RHIC, we use the Collins fragmentation functions [20] extracted from a combined fit to the experimental data from HERMES, COMPASS and BELLE collaborations. We use CTEQ5L parton distributions [28] and Kretzer unpolarized fragmentation function obtained in [29].
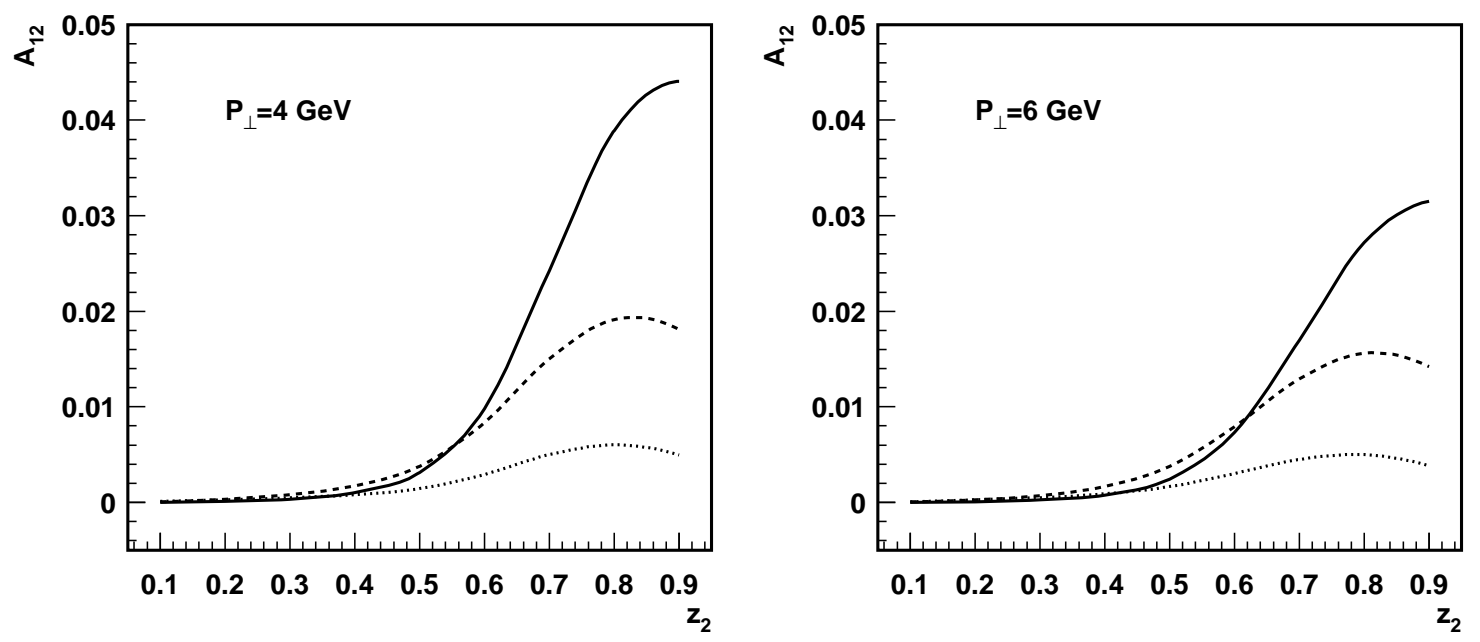

FIG. 3: Azimuthal asymmetry ratios $A_{12}\left(y_{1}, y_{2}, P_{\perp}, z_{1}, z_{2}\right)$ (defined in Eq. (28)) of unlike-sign to like-sign pion production in unpolarized proton-proton scattering at RHIC energy $\sqrt{S}=200 \mathrm{GeV}$, as functions of $z_{2}$ with $y_{1}=y_{2}=0, P_{\perp}=4 \mathrm{GeV}$ (left) or $P_{\perp}=6 \mathrm{GeV}$ (right). The curves are: solid $\left(0.7<z_{1}<0.9\right)$, dashed $\left(0.5<z_{1}<0.7\right)$ and dotted $\left(0.3<z_{1}<0.5\right)$. 
In Fig. 3, we plot $A_{12}\left(y_{1}, y_{2}, P_{\perp}, z_{1}, z_{2}\right)$ for the dihadron production in mid-rapidity $y_{1}=$ $y_{2}=0$ region at RHIC energy $\sqrt{S}=200 \mathrm{GeV}$. In the figure on the left, we plot $A_{12}$ as a function of $z_{2}$ at the jet transverse momentum $P_{\perp}=4 \mathrm{GeV}$ with $z_{1}$ integrated from three different ranges $0.3<z_{1}<0.5$ (dotted), $0.5<z_{1}<0.7$ (dashed), and $0.7<z_{1}<0.9$ (solid), respectively. On the right, we present the same plot but with $P_{\perp}=6 \mathrm{GeV}$. We find that the asymmetry $A_{12}$ is largest when both $z_{1}$ and $z_{2}$ become large, same as what has been observed in $e^{+} e^{-}$experiments [5]. On the other hand, $A_{12}$ decreases when increasing $P_{\perp}$. This is also consistent with what BELLE observed if one realizes that $\hat{s} / 4 P_{\perp}^{2}=\sin ^{2} \theta$ in parton $\mathrm{CM}$ frame. Though it has similar features as that in $e^{+} e^{-}$collision, the asymmetry in hadronic collision is smaller. This is due to the fact that there is copious $g g \rightarrow g g$ and $q g \rightarrow q g$ contribution to the azimuthal angle independent cross section, while they do not contribute to the azimuthal dependent part since there is no gluon Collins function. However, the asymmetry is still around several percent and shall be measurable at RHIC.

These results can be extended to general kinematics, for example, in two different rapidity regions: $\left|y_{1}\right| \neq\left|y_{2}\right|$. In this case, although the azimuthal angular dependence is not exactly as $\cos \left(\phi_{1}+\phi_{2}\right)$ in Eq. (23), the Collins fragmentation functions will nevertheless lead to a nonzero mean value of $\left\langle\cos \left(\phi_{1}+\phi_{2}\right)\right\rangle$. This can be seen from the differential cross section expression in Eq. (17). The experimental observation of this nonzero effects can be used as signal of the Collins effects, since the normal fragmentation functions $D\left(z, p_{T}\right)$ will not contribute to a nonzero $\left\langle\cos \left(\phi_{1}+\phi_{2}\right)\right\rangle$. We hope that the future RHIC experiments can carry out these measurements, and provide more information on the Collins fragmentation functions, which will help us to pin down the mechanism for the single spin asymmetry in hadronic collisions as we discussed in the Introduction.

\section{SUMMARY}

In this paper, we have studied the dihadron azimuthal correlation produced nearly backto-back in unpolarized hadron collision, arising from the product of two Collins fragmentation functions. Using the latest Collins fragmentation function extracted from the global analysis of available experimental data, we make predictions for the azimuthal correlation of two-pion production in unpolarized $p p$ collisions at RHIC energies. We find that the feature of the asymmetry is similar to those observed in $e^{+} e^{-}$annihilation. The asymmetry 
parameter $A_{12}$ is sizable in the mid-rapidity region for moderate jet transverse momentum, and could be measurable in the experiments. The experimental study of this process could provide the important information on the size of Collins fragmentation function in hadronic collision, at the same time, it could also be used to test the universality properties of the Collins fragmentation function in different processes.

\section{Acknowledgments}

We thank M. Grosse Perdekamp, J. Qiu and R. Seidl for helpful discussions. This work was supported in part by the U. S. Department of Energy under Grant No. DE-FG0205CH11231. We are grateful to RIKEN, Brookhaven National Laboratory, and the U.S. Department of Energy (Contract No. DE-AC02-98CH10886) for providing the facilities essential for the completion of this work.

[1] For a review, see: U. D'Alesio and F. Murgia, Prog. Part. Nucl. Phys. 61, 394 (2008) arXiv:0712.4328 [hep-ph]].

[2] A. Airapetian et al. [HERMES Collaboration], Phys. Rev. Lett. 94, 012002 (2005) arXiv:hep-ex/0408013.

[3] E. S. Ageev et al. [COMPASS Collaboration], Nucl. Phys. B 765, 31 (2007) arXiv:hep-ex/0610068.

[4] B. I. Abelev et al. [STAR Collaboration], Phys. Rev. Lett. 99, 142003 (2007) arXiv:0705.4629 [hep-ex]].

[5] R. Seidl et al. [Belle Collaboration], Phys. Rev. Lett. 96, 232002 (2006) arXiv:hep-ex/0507063; Phys. Rev. D 78, 032011 (2008) arXiv:0805.2975 [hep-ex]].

[6] D. W. Sivers, Phys. Rev. D 41, 83 (1990); Phys. Rev. D 43, 261 (1991).

[7] J. C. Collins, Nucl. Phys. B 396, 161 (1993).

[8] J. C. Collins, Phys. Lett. B 536, 43 (2002). D. Boer et al., Nucl. Phys. B 667, 201 (2003); Z. B. Kang and J. W. Qiu, Phys. Rev. Lett. 103, 172001 (2009).

[9] C. J. Bomhof, P. J. Mulders and F. Pijlman, Phys. Lett. B 596, 277 (2004); Eur. Phys. J. C 47, 147 (2006); JHEP 0702, 029 (2007); C. J. Bomhof and P. J. Mulders, Nucl. Phys. B 795, 
409 (2008) arXiv:0709.1390 [hep-ph]].

[10] J. W. Qiu, W. Vogelsang and F. Yuan, Phys. Lett. B 650, 373 (2007); Phys. Rev. D 76, 074029 (2007).

[11] W. Vogelsang and F. Yuan, Phys. Rev. D 76, 094013 (2007).

[12] J. Collins and J. W. Qiu, Phys. Rev. D 75, 114014 (2007); J. Collins, arXiv:0708.4410 [hep-ph].

[13] A. Bacchetta, C. J. Bomhof, P. J. Mulders and F. Pijlman, Phys. Rev. D 72, 034030 (2005) arXiv:hep-ph/0505268.

[14] A. Metz, Phys. Lett. B 549, 139 (2002); J. C. Collins and A. Metz, Phys. Rev. Lett. 93, 252001 (2004) arXiv:hep-ph/0408249]; S. Meissner and A. Metz, Phys. Rev. Lett. 102, 172003 (2009) arXiv:0812.3783 [hep-ph]].

[15] L. P. Gamberg, A. Mukherjee and P. J. Mulders, Phys. Rev. D 77, 114026 (2008). arXiv:0803.2632 [hep-ph]];

[16] F. Yuan and J. Zhou, Phys. Rev. Lett. 103, 052001 (2009) arXiv:0903.4680 [hep-ph]].

[17] F. Yuan, Phys. Rev. Lett. 100, 032003 (2008) arXiv:0709.3272 [hep-ph]]; Phys. Rev. D 77, 074019 (2008) arXiv:0801.3441 [hep-ph]].

[18] J. P. Ralston and D. E. Soper, Nucl. Phys. B 152, 109 (1979); R. L. Jaffe and X. D. Ji, Phys. Rev. Lett. 67, 552 (1991); Nucl. Phys. B 375, 527 (1992).

[19] D. Boer, Nucl. Phys. B 806, 23 (2009) [arXiv:0804.2408 [hep-ph]].

[20] M. Anselmino, M. Boglione, U. D’Alesio, A. Kotzinian, F. Murgia, A. Prokudin and C. Turk, Phys. Rev. D 75, 054032 (2007) arXiv:hep-ph/0701006.

[21] P. J. Mulders and R. D. Tangerman, Nucl. Phys. B 461, 197 (1996) [Erratum-ibid. B 484, 538 (1997)] arXiv:hep-ph/9510301.

[22] D. Boer and P. J. Mulders, Phys. Rev. D 57, 5780 (1998) arXiv:hep-ph/9711485.

[23] D. Boer, S. J. Brodsky and D. S. Hwang, Phys. Rev. D 67, 054003 (2003) arXiv:hep-ph/0211110.

[24] D. Boer, P. J. Mulders and C. Pisano, Phys. Lett. B 660, 360 (2008) arXiv:0712.0777 [hepph]; arXiv:0909.4652 [hep-ph].

[25] Z. Lu and I. Schmidt, Phys. Rev. D 78, 034041 (2008) arXiv:0805.4006 [hep-ph]].

[26] A. Bacchetta, M. Diehl, K. Goeke, A. Metz, P. J. Mulders and M. Schlegel, JHEP 0702, 093 (2007) arXiv:hep-ph/0611265.

[27] J. F. Owens, Rev. Mod. Phys. 59, 465 (1987). 
[28] H. L. Lai et al. [CTEQ Collaboration], Eur. Phys. J. C 12, 375 (2000) arXiv:hep-ph/9903282].

[29] S. Kretzer, Phys. Rev. D 62, 054001 (2000) arXiv:hep-ph/0003177. 